\title{
A Combined Color, Texture and Edge Features Based Approach for Identification and Classification of Indian Medicinal Plants
}

\author{
Basavaraj S. Anami \\ Principal \\ K.L.E.Institute of Technology, \\ Hubli-580030, Karnataka,India
}

\author{
Suvarna S. Nandyal \\ Research Scholar \\ PDA College of Engineering, \\ Gulbarga-585102, India
}

\author{
A. Govardhan \\ Principal \\ JNTUH College of Engineering \\ KarimNagar-505501, AP, India
}

\begin{abstract}
This paper presents a method for identification and classification of images of medicinal plants such as herbs, shrubs and trees based on color and texture feature using SVM and neural network classifier. The tribal people in India classify plants according to their medicinal values. In the system of medicine called Ayurveda, identification of medicinal plants is considered an important activity in the preparation of herbal medicines. Ayurveda medicines have become alternate for allopathic medicine. Hence, leveraging technology in automatic identification and classification of medicinal plants has become essential. Plant species belonging to different classes such as Papaya, Neem, Tulasi, Aloe and Garlic are considered in this work. This paper presents edge and color descriptors that have low-dimension, effective and simple. In addition, the rotation invariant texture descriptors namely, directional difference and the gradient histogram are used. These features are obtained from 900 images of medicinal plants and used to train and test the image samples of three classes with SVM and radial basis exact fit neural network (RBENN). The classification accuracies for color, edge texture features are $74 \%$ and $80 \%$ respectively. The accuracy is improved to $90 \%$ with combined color and texture features. The results are encouraging for tree image plants than herbs and shrubs due to distinguishing feature of stem.
\end{abstract}

\section{Keywords}

Medicina Plant, Edge histogram, Color histogram, Edge direction, SVM classifier, RBENN classifier.

\section{INTRODUCTION}

Medicinal plants form the backbone of a system of medicine called Ayurveda and is useful in the treatment of certain chronic diseases. Ayurveda is considered a form of alternative to allopathic medicine in the world. This Indian system of medicine has rich history. Ancient epigraphic literature speaks of its strength. Ayurveda certainly brings substantial revenue to India by foreign exchange through export of Ayurvedic medicines, because of many countries inclining towards this system of medicine. Considerable depletion in the population of certain species of medicinal plants, we need to grow these plants in India. This rejuvenation work requires easy recognition and classification of medicinal plants. It is necessary to make people realize the importance of medicinal plants before their extinction. It is important for Ayurveda practitioners and also traditional botanists to know how to identify and classify the medicinal plants through computers. Medicinal plants are classified based on internal and external features. The external features of plants are helpful in their identification. According to the plants taxonomy, we find classification of plants based on the shapes of their leaves and flowers. But plant classification based on color histogram, edge direction, edge histogram is not being attempted by human beings. In this connection, a literature survey is carried out to find the state-of-the-art.

Some researchers have studied the classification process based on hierarchical and other methods. The medicinal plants' classification based on parts, leaves, flowers and stems etc. has shown significant results. A color and texture features based recognition is found in [15][9][2]. The summary of the latest research activities in this field are given in [6][7]. We find the segmentation and histogram generation using the HSV color space and an analysis based on visual perception of the variation in Hue, Saturation and Intensity values of an image pixel in image retrieval [13]. Color-texture classification with color histogram and local binary pattern is used to provide robust pattern-related information. Color histograms contain very discriminative color information [12]. It is proposed that color histograms have identifiable features that relate to a precise mathematical way to scene properties. Object color and illumination color features are the most obvious properties that are related to object color and illumination [3]. SpatialChromatic Histogram ( $\mathrm{SCH}$ ), a novel method for content based image retrieval using Spatial Chromaticity Histogram $(\mathrm{SCH})$ is used in [11]. It synthesizes few values of information about the location of pixels having the same color and their arrangement within the image. Some researchers have tried to use edge histogram descriptor for MPEG-7 for image matching by global and semi-local edge histograms from the local histogram bins. Efforts have also been made for retrieval technique that combines color and edge features for image indexing. They have used the perceptually uniform HSV (Hue, Saturation and Value) color space for color histograms and $\mathrm{YCrCb}$ (luminance/red chrominance/blue chrominance) color space for edge histograms with different distance measures. The work describes 4-bin edge histogram to represent the strength of edge in four directions 0 , $45^{\circ}, 90^{\circ}, 135^{\circ}$.Image gradient was computed by using Prewitt 
operator. It is observed that combined feature gives the better retrieval efficiency [5]. Some work is carried out for weed classification based on color. Color filtering algorithm is implemented as a preprocessing task involving the technique known as the extraction of green color [9]. Some researcher have described plant classification based on leaves shape, color and texture [15]. An integrated method is proposed for color image retrieval by combining features. The performance of image retrieval system is improved by integrating features, color histogram, edge histogram and edge direction feature. The method is tested on general purpose image database and found efficient in retrieval performance [10]. Performance comparison of different classifiers and Support Vector Machine is fairly given in [1]. It is found that the classification accuracy is good by combining color histogram, color coherence vectors and edge histogram. The classification of plants is done mainly based on color and edge features of stem parts. To the best knowledge of authors, no work on identification and classification of Indian medicinal plants is cited. Hence, this is the motivation for the present work on medicinal plant recognition based on the concept of color and edge histograms.

A tree is a woody, perennial plant with a single main stem, generally branching at some distance from the ground and possessing a more or less distinct, elevated crown. The shrub is a loose descriptive term for a woody plant which produces multiple stems, shoots or branches from its base but does not have a distinct single trunk. Herb is strictly, a plant without persistent stems above ground. More generally, any non-woody plant is termed as a herb. Since the color varies from plant to plant and trees and herbs have different color distributions. There is also variation in the branching patterns of trees, herbs and shrubs. Further, a plant with respect to structural appearance is grouped into herb, shrub and tree. These features are captured by color and edge direction information. The work finds applications in Ayurveda practitioners, people working in herbal pharmaceutical industry, botany students and for common people in the identification of herbal plants. The present work has considered images of three classes of medicinal plants. The images of different classes are subjected to preprocessing, color histogram, edge histogram and edge direction histogram features are extracted and classified using Support Vector Machine (SVM) and Radial Basis Exact fit (RBENN)neural network. The developed SVM and RBENN classifiers have given promising results in classifying plants as herbs, shrubs and trees.

The paper is organized into six sections. Section two gives image acquisition and details of the proposed methodology. Feature extraction is presented in section three. Section four describes classification of medicinal plants using SVM and neural network classifier. The results and discussions are given in section five. Section six gives conclusion of the work.

\section{PROPOSED METHODOLOGY}

We have collected the medicinal plant images from different herbarium and farms. The medicinal plants samples are acquired from farms in Gulbarga, herbal gardens, M.M. Joshi park, Budinal Forest and Ayurved college in Hubli, in the state of Karnataka, India.

\subsection{Image Acquisition}

We have obtained 1200 images of medicinal plants using the color Digital Camera NIKON, COOLPIX, having resolution of 8 Mega pixels and mounted on a tripod stand. The distance between the camera and the medicinal plants is kept constant and fixed to 40 centimeters. The views in all the eight directions, namely, right, left, top, bottom, and other diagonals are obtained. The images of plants are captured in such a way that the plant trunk and leafy portion are more significant with clear difference in color and edge pattern.

\subsection{Image Samples}

The images of different medicinal plants are considered in this work. The botanical names, regional names in the brackets, of the plants are Cocos Nucifera(Tengu),Azadirachta Indica(Bevu), Carica Papaya(Papaya), Cathranthus Roseus(SadaMallige), Allium Sativum(Bellulli), Ocimum Sanctum(Tulasi), Calotropis Gigantea(Ekki), Aloe Vera(Laval sara) etc. The sample images of medicinal plants are shown in Figure 1. A total of 900 images, 300 images of each type, namely, trees, herbs and shrubs, are considered for training the classifier. Another set of 100 images of each class are used for testing the classifier. The climbers and creepers are not included in the work.

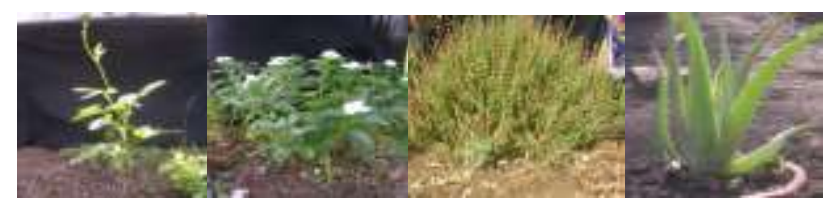

(a)

(b)

(c)

(d)

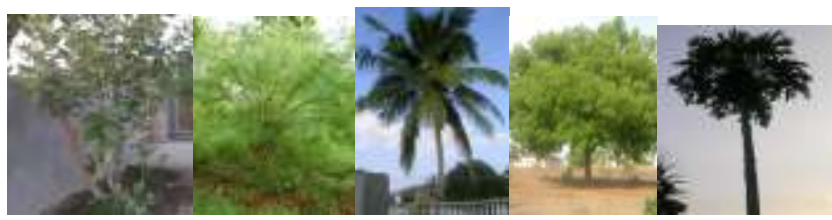

(e)

(f)

(g)

(h)

(i)

Figure 1: Images of medicinal plant (a) VignaunguiCulata (b)Catharanthus Roseus(c)OcimumSanctum (d) AloeVera (e) Calotropis Gigantea (f)NeriumIndica (g)CocosNucifera (h) AzadirachitaIndica (i) Carica papaya

\subsection{Devised Methodology}

The methodology gives the classification of images of medicinal plants by Support Vector Machine (SVM) and neural network classifier using color and texture. The different stages of the methodology are given in Figure 2. The images of medicinal plants are filtered by Gaussian filter to remove any noise introduced at the time acquisition of images. The color, edge and edge direction histograms' features are extracted from the whole images with background. The images of medicinal plants are classified based on morphological and structural appearances. Each medicinal plant is characterized by certain geometrical properties through which they are identified and classified in the real world. We have adopted the following philosophy in the classification. The medicinal plants are classified as herbs, shrubs and trees based on the edge or skeleton feature of stem and leafy mass. The medicinal plant with woody color and single 
stem is considered as tree. A medicinal plant with non woody color with small stem is considered as herb. The shrubs have certain branching pattern or sometimes the leaves shooting from base itself. Figure 3 gives the classification tree of plant species.

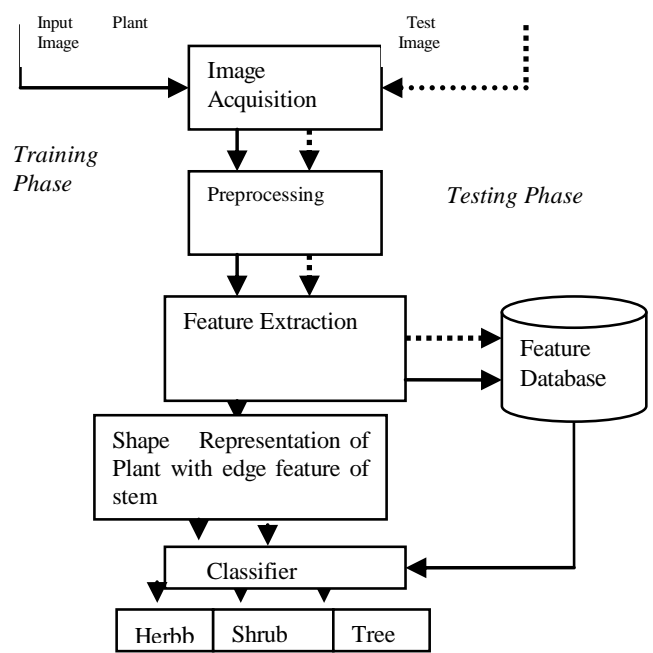

Figure 2: Stages in the developed methodology

These features are extracted with color and edge direction histogram. Since RGB color space does not give the required discrimination in the features, we have used $\mathrm{HSV}$ and $\mathrm{YCbCr}$ color spaces for obtaining the color histogram. The edge information present in the plant image is obtained using edge and edge direction histogram. We have used $\mathrm{YCbCr}$ color space for computing the edge component. These features are used to train a SVM classifier. The results are compared with neural network classifier.

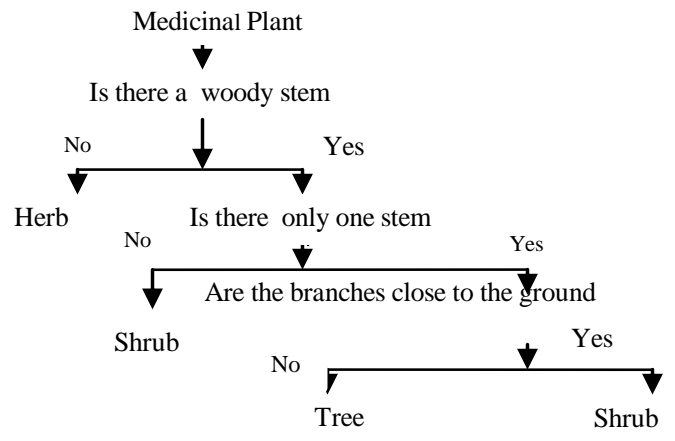

Figure 3: Medicinal plants classification tree

\section{FEATURE EXTRACTION}

The features, namely, color histogram, edge histogram, edge direction histogram are extracted using Sobel operator. The details are given as under.

\subsection{Color histogram}

Usually the color images are converted to gray scale for computational reasons and also interest in the intensity values of the pixels in the given image. In case recognition based on other contexts such as shape or texture, color information is not needed. However, when color features are needed, the captured images are considered. Hence we have used color images for processing. Color histogram is obtained for each class of images. Color histogram helps for finding color distribution of pixels in a plant image. Since color is not uniform over the images, we have transformed the images to other color spaces. Color histogram gives the variance in color in the given image. The images are converted from $\mathrm{RGB}$ color space to $\mathrm{HSV}$ and $\mathrm{YCbCr}$ color spaces, so that variance in color becomes observable well. In HSV color space, $\mathrm{H}$ is the hue which describes the actual wavelength of the color. As depicted in Figure 4, the variation of green color of leafy part becomes distinguishable. The color histogram for plant image is constructed by counting the number of pixels of each color as shown in Figure 4(d)4(e).Color histogram feature value in both the color spaces are shown in Table 1.It is observed the HSV color space gives more significant color distribution values for classification of plants as herbs, shrubs and trees than $\mathrm{YCbCr}$ color space The color feature extraction is given in Algorithm1. (a)

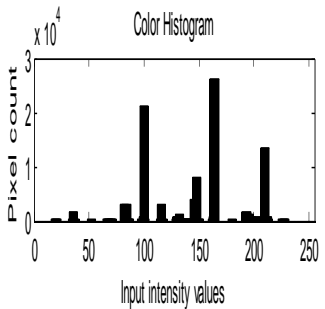

(d)

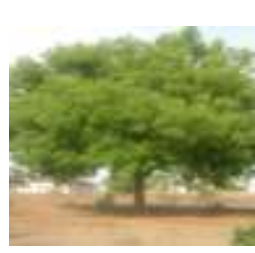

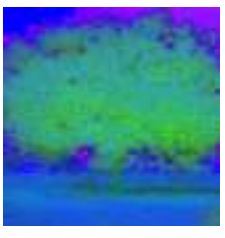

(b)

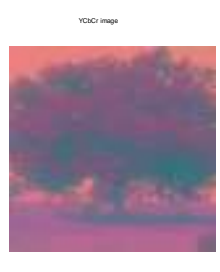

(c)

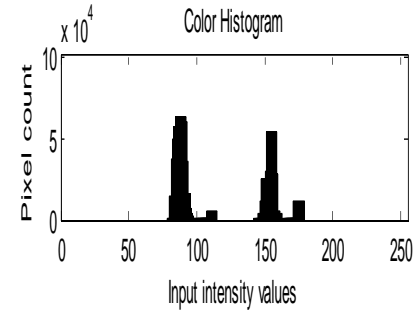

(e)

Figure.4: (a)AzardichataIndica(Neem)tree (b)(c) $H S V \& Y C_{b} C_{r}$ color transformed image $(d)(e)$ Color histogram of $\mathrm{HSV} \& \mathrm{YC}_{b} \mathrm{C}_{r}$ color space image.

\section{Algorithm 1: Color Feature Extraction}

Input: RGB color image of Medicinal plant

Output: Color histogram

Start:

Step 1: Read the input plant image in RGB color space.

Step 2: Convert it into $\mathrm{HSV}$ and $\mathrm{YC}_{\mathrm{b}} \mathrm{C}_{\mathrm{r}}$ color space.

Step 3: Extract the color features $\operatorname{Hue}(\mathrm{H})$, Saturation(S), Intensity value(V), luminanace(Y), blue

Chrominance $\left(\mathrm{C}_{\mathrm{b}}\right)$, red chrominance $\left(\mathrm{C}_{\mathrm{r}}\right)$ components.

Step 4: Use Hue, Saturation and Intensity bins and obtain the histogram. 
Step 5: Similarly ,use Luminance, Chroma component bins and obtain the color histogram.

Step 6: Normalize the color histogram.

End

\subsection{Edge Histogram Texture Feature}

Edge Histogram (EH) is used for extracting textural features of stem and leaf portion of a medicinal plant. The distribution of edges of a medicinal plant serves as a good texture signature. It is also useful in image matching in the absence of any homogeneous texture. The plants exhibit heterogeneous texture features both in leafy and stem portions. Hence edge histogram is chosen to find the given plant shape. Since edge detection is used to characterize the plant boundaries, it is considered an important stage in feature extraction and classification. Edge feature represents the plant boundary and is used for finding the plant shape. It is used for segmentation of plant image from the background. Initially, an image in RGB color space is converted into $\mathrm{YCbCr}$ color space. The luma components, blue-difference chroma componenst and red-difference chroma components of the given plant are captured through $\mathrm{Y}^{\prime}, \mathrm{Cb}$ and $\mathrm{Cr}$ respectively. Filter coefficients used in the work are given in Figure 5 and are used as edge filters in five directions. The edge histogram is obtained by computing the gradient of pixels, that is maximum rate of change of plant image at coordinates(x,y), in the five directions with a threshold value of 100 as given equation (1). We have applied edge filter with different thresholds on the same image. This gives us information on the different gradations on edges of the plant image. We used five different thresholds (Figure 7).When a low threshold was used the gentle edges of leafy mass were detected. As the threshold increases only the more definite edges were detected. The edges of the stem/trunk were detected in low threshold values. We combined each of these images to form a combined image. Pixels that correspond to gentle edges have given a low gray scale value and higher values were given to images of sharper edges of leafy mass.

$$
\begin{aligned}
& \Theta=\arctan \left(\frac{G_{y}}{G_{x}}\right) \text { where } G_{x} \text { and } G_{y} \text { are gradient vector } \\
& \text { in } x \text { and } y \text { direction } \\
& \text { a. }\left[\begin{array}{rr}
1 & -1 \\
1 & -1
\end{array}\right] \text { b. }\left[\begin{array}{rr}
1 & 1 \\
-1 & -1
\end{array}\right] \text { c. }\left[\begin{array}{rr}
\sqrt{2} & 0 \\
0 & -\sqrt{2}
\end{array}\right] \text { d. }\left[\begin{array}{rr}
0 & \sqrt{2} \\
-\sqrt{2} & 0
\end{array}\right] \text { e. }\left[\begin{array}{rr}
2 & -2 \\
-2 & 2
\end{array}\right]
\end{aligned}
$$

Figure 5: (a) Horizontal edge filter (b) Vertical edge filter (c) Diagonal $\left(45^{\circ}\right)$ edge filter (d) Diagonal $\left(135^{\circ}\right)$ edge filter (e) Nondiagonal (isotropic) edge filter

\subsection{Edge Direction HistogramTexture Feature}

The edges of each input plant image are grouped into five classes, vertical, horizontal, 450- diagonal, 1350- diagonal and isotropic (non-directional) based on strengths of directional edges. The sobel operator helps in calculating the edges and direction of edges. The histogram for each of the images of medicinal plants represents the frequencies of occurrences of the five classes of edges in the corresponding images. These directions are determined for each image and an edge mask is formed with threshold value of 100. The edge pixels are characterized by the magnitudes of gradients greater than 100 . The Edge Direction Histogram (EDH) uses the sobel operator to capture the spatial distribution of edges in the six directions with filter mask shown in Figure 6. Edge histogram feature values for three classes of plants is shown in Table 1 .

$$
X=\left[\begin{array}{lrr}
1 & 2 & 1 \\
0 & 0 & 0 \\
-1 & -2 & -1
\end{array}\right] \quad Y=\left[\begin{array}{ccc}
1 & 0 & -1 \\
2 & 0 & -2 \\
1 & 0 & -1
\end{array}\right]
$$

Figure 6: Sobel operator in $\mathrm{X}$ and $\mathrm{Y}$ direction

The edge histogram has eight bins corresponding to the Sobel filters to count the number of edge pixels in eight directions. Edge histogram is normalized with respect to the image size. Figure 8 shows the combined edges computed in horizontal direction for few sample plants. The number of edge pixels in six directions $(0 \mathrm{o}, 45 \mathrm{o},-45 \mathrm{o}, 90 \mathrm{o}, 135 \mathrm{o}, 180 \mathrm{o})$ are shown in Figure 9. The texture feature extraction using edge direction histogram is given in Algorithm 2.

Table 1: Color histogram and edge histogram feature values

\begin{tabular}{|c|c|c|c|c|c|c|c|c|c|c|}
\hline \multirow{2}{*}{$\begin{array}{c}\text { Sl. } \\
\text { No. }\end{array}$} & \multirow{2}{*}{ Plant Species name } & \multicolumn{3}{|c|}{$\mathrm{CH}$} & \multicolumn{4}{c|}{ EDH } & \multicolumn{4}{c|}{$\begin{array}{l}\text { Plant } \\
\text { class }\end{array}$} \\
\cline { 3 - 12 } & & $\mathrm{HSV}$ & $\mathrm{YC}_{\mathrm{b}} \mathrm{C}_{\mathrm{r}}$ & $0^{\circ}$ & $45^{\circ}$ & $-45^{\circ}$ & $90^{\circ}$ & $135^{\circ}$ & $180^{\circ}$ & \\
\hline 1. & Azadirachita Indica & 108.28 & 138.63 & 0.0189 & 0.0098 & 0.0250 & 0.0114 & 0.0071 & 0.0112 & Tree \\
\hline 2. & CaricaPapaya & 108.32 & 120.87 & 0.0150 & 0.0063 & 0.0205 & 0.0147 & 0.0015 & 0.0049 & Tree \\
\hline 3. & CatharanthusRoseus & 99.65 & 121.73 & 0.0294 & 0.0187 & 0.0345 & 0.0106 & 0.0080 & 0.0078 & Herb \\
\hline 4. & HibiscusCannabinus & 92.38 & 125.34 & 0.0203 & 0.0121 & 0.0336 & 0.0087 & 0.0041 & 0.0035 & Herb \\
\hline 5. & Aloevera & 102.30 & 123.59 & 0.0166 & 0.0107 & 0.0201 & 0.0048 & 0.0066 & 0.0096 & Shrub \\
\hline 6. & OcimumSanctum & 102.89 & 121.73 & 0.0183 & 0.0099 & 0.0242 & 0.0103 & 0.0076 & 0.0128 & Shrub \\
\hline
\end{tabular}



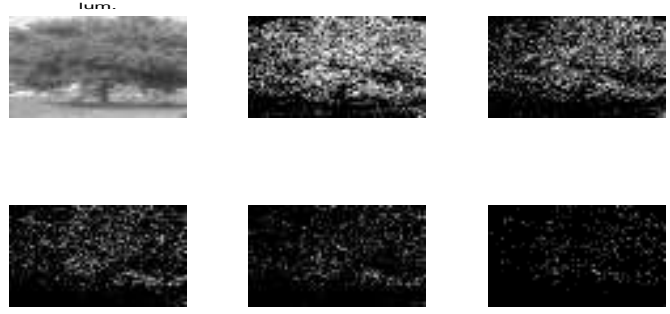

Figure 7: Effect of threshold on edge histogram, with threshold values $=15,25,35,45$ and 55 .
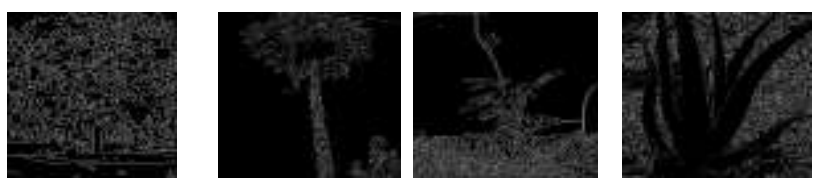

Figure 8:Edges of (a) Neem (b) Papaya (c) Vitex Negundia (d)Aloe vera.

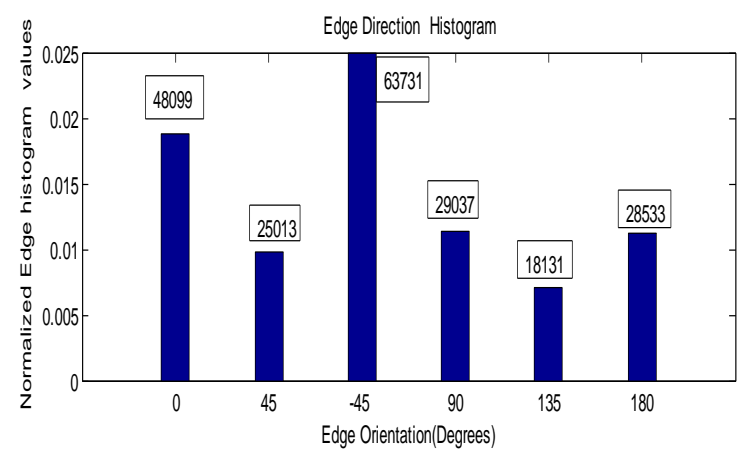

Figure 9: Edge histogram plot of Neem plant image

\section{Algorithm 2:Texture Feature Extraction}

Input: RGB color image

Output: Edge and Edge direction histogram of the plant image

Start:

Step 1: Read the RGB image of plant.

Step 2: Convert it into $\mathrm{YC}_{\mathrm{b}} \mathrm{C}_{\mathrm{r}}$ color space.

Step 3: Extract the luminance(Y) component of the image.

Step 4: Obtain the edge and edge direction histogram using filter mask coefficients.

Step 5: Compute the gradient in the respective direction of the filter mask.

Step 6: Compare the gradient value with the threshold.

Step 7: If (Gradient value > threshold)

then it is an edge and draw the edge map.

Step 8: Normalize the edge histogram and edge direction histogram.

End

\section{VIABILITY OF CLASSIFIERS}

We have tried two types of models, namely, support vector machine (SVM) and artificial neural network (ANN). In case of SVM, three classes are used. A multi-class problem is reduced to three 2-class, pair wise multiclass scheme. We have used three, 2-class support vector machine (SVM) for classification of medicinal plants. Radial basis function (RBF) with scaling factor 1 is applied. The input training samples and labels of training samples are passed as parameters with 2-class SVM classifier. Another classifier used is radial basis neural network. The network architecture consists of two layers. A hidden radial basis layer consists of 256 neurons, corresponding to number of input features. The output layer consists of three neurons, corresponding to the number of output classes. The performance of the work is compared with Radial Basis Exact fit (RBENN) classifier.

Radial basis network with exact fit function produces a network with zero error on training vectors. Hence, it is widely used in many automated applications. The trained feature vectors are validated and tested with images of plants of different classes. The output value of ANN is according to how close the input vector is to each neuron's weight vector. A radial basis neuron with a weight vector close to the input feature vector produces a value nearer to unity. If a neuron has an output of 1 , the output weights in the second layer pass their values to the linear neurons in the second layer. The output of linear layer is 1 for one input feature vector only based on the distance between input and weight matrix and for all other vectors the outputs are 0 's. The output target values are set to $\mathrm{T}\left(\begin{array}{lll}1 & 0 & 0\end{array}\right), \mathrm{T}\left(\begin{array}{lll}0 & 1 & 0\end{array}\right)$ and $\mathrm{T}\left(\begin{array}{l}0 \\ 0\end{array}\right.$ 0 1) for herbs, shrubs and trees respectively. The image samples are divided into training and testing data sets. We have used 300 images of each class for training and 100 images for testing. The network is validated with $20 \%$ of the images from the training set.

\section{RESULTS AND DISCUSSION}

The color histogram and edge histogram features are extracted from medicinal plant images of different classes under natural lighting condition and used for recognition and classification. The features are trained with SVM classifier and neural network classifier.

\subsection{Recognition and classification using SVM and Artificial Neural Network classifiers}

The classification accuracies of the samples comprising herbs, shrubs and trees is computed using expression (2)

$$
\text { Classification Rate }(\%)=\frac{\text { Number of correctlyclassifiedsamples }}{\text { Total Number of samples }} \text { X100.(2) }
$$

\subsubsection{Based on Color Feature}

The color histogram feature listed in Table 1 are extracted using Algorithm 1. Two-class SVM classifier is trained with 256 color distribution values for each color space, HSV and $\mathrm{YCbCr}$. Hence, a SVM classifier is constructed with 256 training samples and 3 output classes. The SVM classifier uses a decision function to group the 256 input training samples into 
three class labels. The labels are generated with respect to number of support vectors. Hence, based on the color, the color histogram for tree images shows a clear valley and is spread between 0 and 255 .

From the experimentation, it is clear that trees are fairly classified since trees have well defined color distribution values. The HSI color space allows access to the hue and intensity information directly from the image and it provides more uniform perceptual color values similar to human visualization. Hence, classification accuracy with HSI components is greater than $\mathrm{YCbCr}$ components. From the Table 1,we have observed overlapped values of $\mathrm{YCbCr}$ components for different medicinal plant classes. Hence, we have given in Figure 11 only the percentage of classification accuracy of medicinal plant classes in HSI color space. It is also observed that the accuracy depends upon illumination intensities. The average classification accuracy of $74 \%$ is obtained with color features. Other than color, the edge features of plants are also helpful in distinguishing the herbs, shrubs and trees. Hence we have attempted recognition with edge histogram and edge direction histogram as texture features.

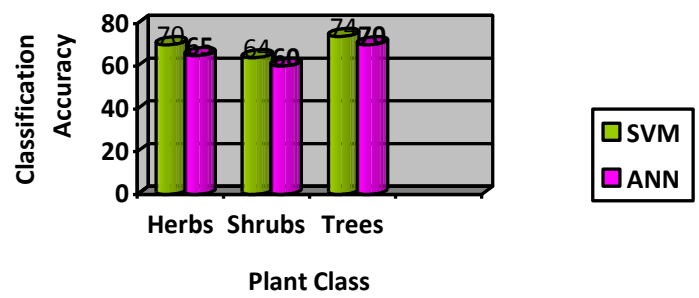

Figure 11: Color histogram based classification accuracy.

\subsubsection{Edge based texture features}

The training sample consists of 5 edge histograms and 6 edge direction histograms as features amounting to a total of 11 features. The edge direction texture feature performed well in extracting trunk parts from images of different medicinal plants images. The edge information gives the most discriminating feature for herb,shrub and tree recognition and classification. Hence, the edge direction histogram feature gave high classification accuracy for both the classifier. Figure 12 shows, the percentage accuracy of recognition for herbs, shrubs and trees images are $[84.33 \%, 80.67 \%], \quad[72.7 \%, 67.44]$ and [88.23\%,84.59\%],respectively with SVM classifier and neural network classifier. It is revealed that the classification accuracy for trees is good. But the methodology is vulnerable to herbs and shrubs, as these plants do not have a distinguished stem part. The recognition and classification accuracy is improved when we have used edge information for the same set of plant image species. This is because each plant is identified mainly based on the edge and branching pattern of stem/trunk and leafy mass. Hence edge texture becomes the more discriminating feature for classification. The average accuracies have increased to $74 \%$ to $80 \%$ using texture information. Therefore, edge texture features are more suitable when different plants exhibits same color.

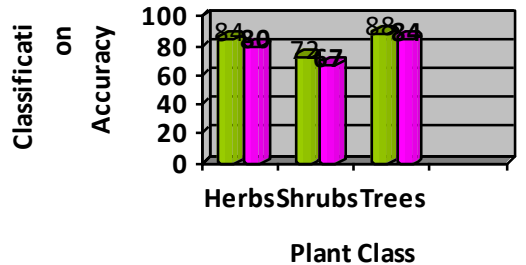

$\square$ SVM
$\square$ ANN

Figure 12: Edge and Edge direction texture features classification accuracy.

\subsubsection{Combined color and texture features}

Experiments are performed on combined color, edge histogram $(\mathrm{EH})$ and edge direction histogram(EDH) texture features using SVM and ANN classifiers. The number of input features is 267. The output classes are 3 . Figure 13 gives the classification accuracies observed for the medicinal plant images of the three classes. The maximum and minimum classification accuracies observed are $94 \%$ and $70 \%$ with SVM classifier. The maximum and minimum classification accuracies observed are $90 \%$ and $65 \%$ with ANN classifier. The classification accuracy is maximum for trees and minimum for shrubs, with both the types of classifiers. The images of shrubs species do not have edge information and hence the accuracy is suffered. The experimental results have shown that the combined color and edge histogram texture features improved the classification accuracy from $74 \%$ to $90 \%$.

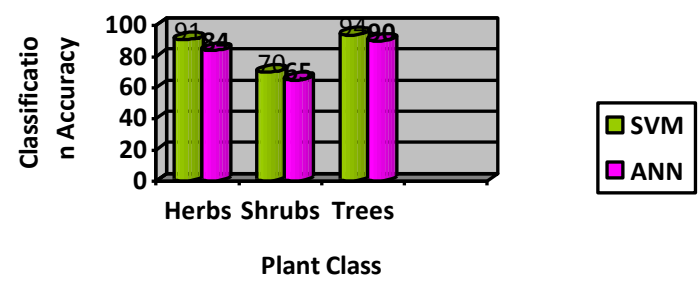

Figure 13: Combined color and texture features classification accuracy

\section{CONCLUSION}

The experimental results shows that trees are fairly classified using the proposed methodology as the edge and color features for trees are clearly distinguishable than herbs and shrubs. Classification based on color histogram feature gives lower accuracy, since majority of the plants have green color. Moreover, the shades change seasonally causing color feature reliability low. Hence, the good results are obtained by the combination of color and texture (edge) features. It is found that classification accuracy is better with SVM classifier than neural network classifier. The methodology has not performed well for the images of herbs and shrubs. This is attributed to lack of edge information of stem portion. The work assists human beings in classification of medicinal plants in the real world and considered an essential task in pharmaceutical industry, Ayurved practitioners and botanists. 


\section{REFERENCES:}

[1] A. Commander Sunil Tyagi, (2008). A Comparative Study of SVM Classifiers and Artificial Neural Networks Application for Rolling Element Bearing Fault Diagnosis using Wavelet Transform Preprocessing, World Academy of Science, Engineering and Technology, Vol. 43,pp 309-317.

[2] B.S.Manjunath,Jens-Rainer Ohm, Vinod V. Vasudevan, and Akio Yamada,(2001). Color and texture descriptors, IEEE Transactions On Circuits And Systems For Video Technology, Vol. 11, No. 6, pp. 703-715.

[3] Carol L. Novak and Steven A Safar,(1992). Anatomy of a color histogram, proceedings of IEEE Computer Society Conference on Computer Vision and Pattern Recognition (CVPR 92),pp. 599-605.

[4] D Shi, L Xu, L Han,(2008). Image retrieval using both color and texture features, The Journal of China Universities of Posts and Telecommunications, Vol 14, pp.94-99.

[5] Dong Kwon Park, Yoon Seok Jeon, Chee Sun Won,(2000). Efficient Use of Local Edge Histogram Descriptor, International Multimedia Conference Proceedings of ACM workshops on Multimedia, pp 51-54.

[6] Dong-cheng Shi, Lan $\underline{\mathrm{X}} \mathrm{u}$ and Ling-yan Han,(2007). Image retrieval using both color and texture feature, The Journal of China Universities of Posts and Telecommunications, Vol.14. pp 94-99.

[7] Dong-cheng Shi, Lan Xu and Ling-yan Han,(2008). Image retrieval using both color and edge histogram, Electronic Imaging and Multimedia Technology, Vol. 6833(2), pp.6833361-6833367.

[8] Justin Domke and Yiannis Aloimonos,(2006).Deformation and Viewpoint Invariant Color Histograms, Proceedings of British Machine Vision Conference(BMVC),Edinburg UK, pp.267-270.
[9] Kamarul Hawari Ghazali, Mohd Marzuki Mustafa, Aini Hussain,(2007).Color image processing of weed classification:A comparision of two Feature Extraction Techniques,Proceedings of the International Conference on Electrical Engineering and Informatics, pp 607-610.

[10] K. Singh, M. Ma, and D.W. Park,(2003). Histogram Approach for Content-based Image Retrieval, Proceedings in Visualization, Imaging, and Image Processing.

[11] L. Cinque, S. Levialdi, A. Pellicanò, K.A. Olsen,(1999). Color-Based Image Retrieval Using Spatial-Chromatic Histograms, IEEE International Conference on Multimedia Computing and Systems (ICMCS'99) - Volume 2, pp. 969.

[12] Matti Pietik.inen, Topi M.enp.. and Jaakko Viertola,(2002). Color texture classification with color histograms and local binary patterns, In Workshop on Texture Analysis in Machine Vision, pp. 109-112

[13] Shamik Sural, Gang Qian and Sakti Pramanik,(2002). Segmentation and histogram generation using the HSV color space for image retrieval, International Conference on Image Processing(ICIP), Vol. 2 , pp. 589-592.

[14] Shengsheng Yu,Chaobing Huang,Jingli Zhou,(2006). Color Image Retrieval Based On Color-Texture-Edge Feature Histogram, International Journal of Image and Graphics ,Vol 6 No 4 pp 583-598.

[15] Xiao-Feng, Wang, De-Shuang, Huang, Ji-Xiang, Dua,HuanXu, LaurentHutte,(2008). Classification of plant leaves with complicated background, Applied Mathematics and Computation, Vol.205 pp.916-926.

[16] Yining Deng, B. S. Manjunath, Charles Kenney, Michael S. Moore, and Hyundoo Shin, (2001).An Efficient Color Representation for Image Retrieval, IEEE transactions on image processing, Vol. 10, No. 1,pp140-147. 